ISSN 1991-8631

Original Paper

http://indexmedicus.afro.who.int

\title{
Effet de la fertilisation au molybdène sur la biomasse et le nombre de nodules de Desmodium intortum à différentes périodes de fauche dans l'Ouest Cameroun
}

\author{
Arsène V. MBOKO ${ }^{1}$, Fernand TENDONKENG ${ }^{2}$, Férence N. E. MATUMUINI ${ }^{1}$, \\ Gilbert T. ZOUGOU ${ }^{1}$, Benoît BOUKILA ${ }^{1}$ et Etienne T. PAMO ${ }^{2 *}$ \\ ${ }^{1}$ Institut National Supérieur d'Agronomie et de Biotechnologie (INSAB), Université des Sciences et Techniques \\ de Masuku, B.P. 941 Franceville, Gabon. \\ ${ }^{2}$ Laboratoire de Nutrition Animale, Département des Productions Animales, FASA, Université de Dschang. \\ B.P. 222 Dschang, Cameroun. \\ "Auteur correspondant, E-mail: pamo_te@yahoo.fr
}

\section{RESUME}

L'effet de la fertilisation au molybdène sur les biomasses et le nombre des nodules de Desmodium intortum a été évalué à différentes périodes de fauche à la Ferme d'Application et de Recherche de l'Université de Dschang en 2011. Un dispositif factoriel comparant quatre doses de molybdène sous forme de molybdate d'ammonium $(0 ; 0,75 ; 1,5$ et $2,25 \mathrm{~kg} \mathrm{Mo} / \mathrm{ha})$ et six périodes de fauches dans des pots de $0,0346 \mathrm{~m}^{2}$ de surface en trois répétitions, soit un total de 72 pots expérimentaux, a été utilisé. A chacune des 6 périodes de collecte des données, les plantes fauchées étaient séparées en deux parties : la partie aérienne et les racines nodulées. Ensuite, les nodules étaient séparés des racines, collectés et comptés. Les nodules des trois pots par niveau de fertilisation et les parties aériennes étaient pesés puis placés dans une étuve ventilée à $60{ }^{\circ} \mathrm{C}$ jusqu'à poids constant pour la détermination des biomasses en $\mathrm{kg}$ MS/ha et $\mathrm{t}$ MS/ha respectivement pour les nodules et les parties aériennes. La fertilisation au molybdène a influencé de manière variable la biomasse foliaire. La valeur la plus élevée (25,1 t MS/ha) a été obtenues avec la dose de $0 \mathrm{~kg} \mathrm{Mo} / \mathrm{ha}$ à la $90^{\mathrm{e}}$ période de fauche ; alors que la plus faible ( $1,07 \mathrm{t} \mathrm{MS/ha)} \mathrm{avec} \mathrm{celle} \mathrm{de} 1,5 \mathrm{~kg} \mathrm{Mo} / \mathrm{ha}$. Une relation significative $(\mathrm{p}<0,01)$ a été établie entre les biomasses foliaires et les périodes de fauche comme le montre le coefficient de régression $\mathrm{R}^{2}=0,51$. La fertilisation au molybdène n'a pas influencé de manière significative $(\mathrm{p}<0,05)$ la biomasse nodulaire de Desmodium intortum quelles que soient les périodes de fauche. La valeur la plus élevée (280 kg MS/ha) a été obtenue avec la dose de $2,25 \mathrm{~kg} \mathrm{Mo} / \mathrm{ha}$ au $90^{\mathrm{e}}$ jour et la plus faible $(9,73 \mathrm{~kg} \mathrm{MS} / \mathrm{ha}$ ) avec celle de 1,5 $\mathrm{kg} \mathrm{Mo} / \mathrm{ha}$ au $30^{\mathrm{e}}$ jour. Des relations entre les biomasses nodulaires et les périodes de fauche $\left(\mathrm{R}^{2}=0,76\right)$ d'une part et entre les biomasses nodulaires et les biomasses foliaires $\left(R^{2}=0,29^{\text {ns }}\right)$ d'autre part ont été observées. La fertilisation au molybdène n'a pas également influencé de manière significative le nombre des nodules de Desmodium intortum quelle que soit la période. De même, les nombres de nodules n'ont pas connu une évolution régulière en fonction des doses de molybdène. Le nombre le plus élevé (338) des nodules a été enregistré au $90^{\mathrm{e}}$ jour avec la dose de $0,75 \mathrm{~kg} \mathrm{Mo} / \mathrm{ha}$, alors que le plus faible $(45,3)$ a été obtenu avec la dose de $0,75 \mathrm{~kg} \mathrm{Mo} / \mathrm{ha}$ au $180^{\mathrm{e}}$ jour. Aucune relation n'a pu être établie entre le nombre de nodules et les périodes de fauche. (C) 2013 International Formulae Group. All rights reserved.

Mots clés : biomasse foliaire, nodules, légumineuse, nodulation, Rhizobium. 


\section{INTRODUCTION}

Dans les zones tropicales, la pauvreté des sols en azote représente un handicap majeur (Sapolin, 2007) à l'augmentation des rendements des cultures. Le coût élevé, et parfois la non disponibilité des engrais chimiques pour y faire face, oblige la recherche d'autres stratégies pour augmenter la fertilité des sols. Parmi les options qui s'offrent, les légumineuses herbacées ou subligneuses semblent être une alternative, du fait de leur capacité d'enrichir rapidement les sols par la fixation symbiotique de l'azote atmosphérique, de leur production d'une biomasse végétale abondante (Berger et al., 1987) et de leur aptitude à lutter contre les adventices (Toure, 2001).

Desmodium intortum, originaire $\mathrm{du}$ centre et du Sud de l'Amérique (Rakotoarison, 2005), est une plante pérenne, rampante et grimpante. Elle est capable de s'enraciner au niveau des nœuds, possède une racine pivotante et de longues tiges. Il a des feuilles pubescentes, ramifiées, souvent de couleur brun-rouge. Comme beaucoup de légumineuses, Desmodium intortum présente un intérêt agronomique car elle contribue à l'enrichissement des sols en azote grâce au rhizobium et constitue un bon précédent cultural pour les céréales (Le Soudeur et Labreuche, 2007). Mais l'optimisation de la production de cet azote biologique est sous la contrainte de plusieurs facteurs environnementaux et nutritionnels (Bambara et Ndakidemi, 2009). C'est le cas de la carence en Molybdène souvent observée dans les sols acides (Bhupal et al., 2009). En effet, le molybdène est un microélément, constitutif de l'enzyme impliquée dans la fixation de l'azote gazeux par les Rhizobiums des légumineuses et de celui impliquée dans la réduction des nitrates en particulier. $\mathrm{Le} \mathrm{NH}_{4}{ }^{+}$ provenant de cette réduction est incorporé dans les squelettes carbonés pour former les acides aminés par le processus d'assimilation de l'azote (Morot-Gaudry, 1997). Une bonne connaissance de telles informations dans les différentes zones écologiques permettraient d'améliorer la contribution des légumineuses en azote du sol par le processus de fixation symbiotique de l'azote dans la nutrition minérale des plantes. C'est pour contribuer á l'amélioration des connaissances sur l'importance de ces phénomènes dans les sols pastoraux de l'Ouest Cameroun que cette étude a été initiée. Elle devrait montrer l'influence du molybdène sur la production foliaire et nodulaire des légumineuses. L'objectif de ce travail était donc d'évaluer la production de biomasse (foliaire et nodulaire) et le nombre de nodules de Desmodium intortum fertilisé au molybdène.

\section{MATERIEL ET METHODES \\ Zone d'étude}

L'étude a été conduite à la Ferme d'Application et de Recherche (FAR) de l'Université de Dschang entre Juin et Novembre 2011. La FAR est située en région Soudano-guinéenne du Cameroun à $05^{\circ} 20^{\prime}$ latitude Nord et $10^{\circ} 03^{\prime}$ longitude Est et à une altitude moyenne de $1410 \mathrm{~m}$. Le climat de la région est équatorial de type Camerounien, modifié par l'altitude. Les températures oscillent entre $10{ }^{\circ} \mathrm{C}$ (juillet - août) et $25{ }^{\circ} \mathrm{C}$ (février) avec une insolation annuelle de 1800 heures et une humidité relative variant entre 40 - 97\%. Les précipitations varient entre 1500 et $2000 \mathrm{~mm}$ par an. La saison sèche va de mi-novembre à mi-mars et la saison des pluies de mi-mars à mi-novembre correspondant à la période de culture dans cette région.

\section{Conduite de l'essai}

L'essai a consisté en la mise en place de la légumineuse fourragère dans des pots de superficie $0,0346 \mathrm{~m}^{2}$ pour l'étude des biomasses et du nombre des nodules.

Un dispositif factoriel $(4 \times 6)$ comparant quatre (4) doses de molybdène sous forme de molybdate d'ammonium ( 0 ; 0,$75 ; 1,5$ et $2,25 \mathrm{~kg} / \mathrm{ha}$ ) et six (6) périodes de fauches, espacées de 30 jours en trois répétitions, soit un total de 72 pots a été utilisé dans cet essai. Les pots remplis de terre ont été placés côte à côte par lot de 18 et par niveau de fertilisation, dans les mêmes conditions de température, d'humidité et d'éclairage. 


\section{Mise en place des éclats de souches}

La préparation des pots expérimentaux a consisté au remplissage de ceux-ci avec de la terre prélevée dans le site expérimental. Des touffes de Desmodium intortum ont été prélevées dans les parcours de la FAR. Ces touffes ont été habillées (ont subi une réduction de taille des racines et des feuilles) à l'aide de ciseaux. Des éclats de souche de deux (2) rejets obtenus par division manuelle ont été repiqués au centre de chaque pot, à une profondeur de $4 \mathrm{~cm}$.

\section{Fertilisation et entretien des plantes}

Un engrais phosphaté sous forme de superphosphate simple (100 kg par hectare, soit $0,346 \mathrm{~g}$ par unité expérimentale) à $21 \%$ et un engrais potassique sous forme de chlorure de potassium (35 kg par hectare soit $0,121 \mathrm{~g}$ par unité expérimentale), a été appliqué sur l'ensemble des 72 pots comme engrais de fond. Un mois après la mise en place d'éclats de souches de la légumineuse et après la première levée, un désherbage manuel des pots a été pratiqué. Ensuite, les pots ont été fertilisés au molybdène en une seule fois selon le plan expérimental.

Afin d'assurer une homogénéité d'application et compte tenu des quantités très faibles de molybdène, chaque dose à apporter par niveau de fertilisation au molybdène a été dissous dans $180 \mathrm{ml}$ d'eau de façon à apporter exactement $10 \mathrm{ml}$ de solution au pied de chaque plante à l'aide d'une seringue de 10 cc. L'entretien a consisté au désherbage des pots et au nettoyage des espaces entre les pots et des allées entre les blocs de pots.

\section{Collectes des données}

Un mois après la fertilisation au molybdène et deux mois après la mise en place de Desmodium intortum, trois (3) pots par niveau de fertilisation pour un total de 12 pots étaient prélevés au hasard et ceci se répétait tous les 30 jours (correspondant à la période de fauche) pour l'évaluation des biomasses et du nombre des nodules. A chacune des 6 périodes de collecte des données, les plantes étaient séparées en deux : la partie aérienne et les racines nodulées. Ensuite, les nodules étaient séparés des racines par dépôt des pots dans un bassin contenant de l'eau. L'eau par la suite faisait dissoudre lentement la terre qui se dégageait des racines. Les nodules étaient alors collectés et comptés. Les nodules des trois pots par niveau de fertilisation et les parties aériennes étaient pesés puis séchés dans une étuve ventilée de marque Gallenkamp à $60{ }^{\circ} \mathrm{C}$ jusqu'à poids constant pour la détermination des biomasses en $\mathrm{kg} \mathrm{MS} / \mathrm{ha}$ et $\mathrm{t} \mathrm{MS} / \mathrm{ha}$ respectivement pour les nodules et la partie aérienne.

\section{Analyses statistiques}

Les données sur les biomasses et le nombre des nodules ont subi une transformation logarithmique, $\log _{10}(x+1)$ (Gomez \& Gomez, 1984), à cause des très grandes variations entre les écarts types des moyennes, avant d'être soumises à une analyse de la variance (ANOVA) suivant le Model Linéaire General (MLG). Lorsque les différences existaient, les moyennes étaient séparées par le test de Duncan au seuil de signification 5\% (Steel et Torrie, 1980).

\section{RESULTATS}

Effet de la fertilisation au molybdène sur la biomasse foliaire de Desmodium intortum en fonction de la période de fauche

Les variations de la biomasse foliaire de Desmodium intortum en fonction des doses de molybdène aux différentes périodes de fauche montrent que la fertilisation au molybdène a influencé de manière variable la biomasse foliaire (Tableau 1). A la première période de fauche, les biomasses foliaires ont baissé avec les niveaux croissants de molybdène jusqu'à la dose de $1,5 \mathrm{~kg} \mathrm{Mo} / \mathrm{ha}$ avant d'augmenter légèrement avec la dose maximale. La biomasse obtenue des plantes non fertilisées a été significativement $(\mathrm{p}<0,05)$ plus élevée que celle observée avec la dose de $1,5 \mathrm{~kg} \mathrm{Mo} / \mathrm{ha}$ mais comparable $(\mathrm{p}>0,05)$ à celles observées avec les doses de 0,75 et 2,25 $\mathrm{kg} \mathrm{Mo/ha} \mathrm{qui} \mathrm{ont} \mathrm{par} \mathrm{ailleurs} \mathrm{été} \mathrm{comparables}$ entre elles.

$\mathrm{Au} 60^{\mathrm{e}}$ jour de fauche, les valeurs des biomasses ont augmenté avec l'application des doses de 0,75 et $1,5 \mathrm{~kg} \mathrm{Mo} /$ ha avant de baisser avec la dose de 2,25 kg Mo/ha. La 
biomasse obtenue des plantes fertilisées avec la dose de 1,5 kg Mo/ha (6,39 tMS/ha) a été statistiquement comparable à celles obtenues des plantes fertilisées avec les doses de 0,75 et $2,25 \mathrm{~kg} \mathrm{Mo} / \mathrm{ha}$. Mais elle était significativement $(p>0,05)$ supérieure à celle obtenue des plantes non fertilisées. Aux 90, 120,150 et $180^{\mathrm{e}}$ jour de fauche, les valeurs de biomasses ont évolué en dents de scie en fonction des doses de molybdène. Cependant, aucune différence significative $(\mathrm{p}<0,05)$ n'a été observée entre les biomasses foliaires en fonction des doses de fertilisation. Toutefois, les valeurs de biomasses les plus élevées ont été obtenues avec les doses de $0(25,1$ et 23,7$)$, $1,5 \quad(21,8)$ et $2,25 \mathrm{~kg}$ Mo/ha $(17,8)$ respectivement aux périodes de fauche de 90 , 120,150 et $180^{\mathrm{e}}$ jour. Alors que les plus faibles 13,$8 ; 14,4 ; 10,1$ et $10,4 \mathrm{t} \mathrm{MS} /$ ha ont été observées avec les doses de 2,$25 ; 0,75$; 2,25 et $1,5 \mathrm{~kg} \mathrm{Mo} / \mathrm{ha}$ respectivement.

Une relation significative $(\mathrm{p}<0,01)$ a été établie entre les biomasses foliaires et les périodes de fauche comme le montre le coefficient de détermination $\mathrm{R}^{2}$ de l'équation suivante :

$\left(\mathrm{R}^{2}=0,51\right)$

Biomasse foliaire $=0,11 \mathrm{x}$ jour $+1,43$

Effet de la fertilisation au molybdène sur la biomasse nodulaire de Desmodium intortum en fonction de la période de fauche

Des résultats du Tableau 2, il apparait que la fertilisation au molybdène n'a pas influencé de manière significative $(\mathrm{p}<0,05)$ les biomasses nodulaires de Desmodium intortum quelles que soient les périodes de fauche. Aux périodes de fauche de 30,60, 150 et 180 jours, les biomasses ont évolué de manière irrégulière en fonction des différentes doses de fertilisation. Les valeurs de biomasses nodulaires les plus élevées $(50,6 ; 120 ; 118$ et $61,7 \mathrm{~kg} \mathrm{Ms} / \mathrm{ha}$ ) aux périodes de fauche citées ci-dessus ont été obtenues avec les doses de 0,$75 ; 1,5$ et $0 \mathrm{~kg} \mathrm{Mo} / \mathrm{ha}$ respectivement. Les biomasses les plus faibles $(9,73 ; 85,2 ; 94,9$ et $25,2 \mathrm{~kg} \mathrm{Ms} / \mathrm{ha})$ ont été obtenues respectivement avec les doses de 1,$5 ; 2,25$ et $0,75 \mathrm{~kg} \mathrm{Mo} / \mathrm{ha}$. Au $90^{\mathrm{e}}$ jour de fauche, les doses de $0,75,1,5$ et $2,25 \mathrm{~kg} \mathrm{Mo} / \mathrm{ha}$ ont permis une augmentation de la biomasse nodulaire; alors qu'au $120^{\mathrm{e}}$ jour, la biomasse a augmenté jusqu'à $1,5 \mathrm{~kg} \mathrm{Mo} / \mathrm{ha}$ avant de baisser avec l'apport de la dose maximale de fertilisation.

Aucune différence significative $(\mathrm{p}<0,05)$ n'a été cependant observée entre les biomasses en fonction des doses de molybdène quelle que soit la période de fauche considérée. Toutefois, des relations entre les biomasses nodulaires et les périodes de fauche d'une part et entre les biomasses nodulaires et les biomasses foliaires d'autre part ont été établies comme le montrent les équations de régression suivantes:

* Biomasse nodulaire $=4,16 \times$ jours 0,02 xjours $^{2}-75,1, \quad\left(\mathrm{R}^{2}=0,76\right)$

* Biomasse nodulaire $=34,67 \mathrm{Ln}$ (biomasse foliaire) $+21,9, \quad\left(\mathrm{R}^{2}=0,29^{\mathrm{ns}}\right)$.

Effet de la fertilisation au molybdène sur le nombre de nodules de Desmodium intortum en fonction de la période de fauche

Le Tableau 3 présente l'évolution du nombre de nodules de Desmodium intortum aux différentes périodes de fauche en fonction des doses de fertilisation au molybdène. De ce tableau, il ressort que pour l'ensemble des périodes de fauche, le nombre de nodules n'a pas connu une évolution régulière en fonction des différents niveaux de fertilisation au molybdène. Les nombres les plus élevés 163 $\left(30^{\mathrm{e}}\right) ; 262\left(60^{\mathrm{e}}\right) ; 338\left(90^{\mathrm{e}}\right) ; 304\left(120^{\mathrm{e}}\right) ; 225$ $\left(150^{\mathrm{e}}\right) ; 149\left(180^{\mathrm{e}}\right.$ jour $)$ ont été obtenus des plantes fertilisées avec les doses de 0,$75 ; 0$; 0,$75 ; 1,5 ; 0 \mathrm{~kg} \mathrm{Mo} / \mathrm{ha}$ respectivement. Et les plus faibles $58,3\left(30^{\mathrm{e}}\right) ; 148\left(60^{\mathrm{e}}\right) ; 280\left(90^{\mathrm{e}}\right)$; $187\left(120^{\mathrm{e}}\right) ; 98,3\left(150^{\mathrm{e}}\right)$ et 45,3 au $180^{\mathrm{e}}$ jour de fauche ont été observées des plantes fertilisées avec les doses de 1,$5 ; 2,25 ; 1,5 ; 0,75 ; 2,25$ et $0,75 \mathrm{~kg} \mathrm{Mo} / \mathrm{ha}$ respectivement. Aucune différence significative $(p<0,05)$ n'a été observée entre les nombres des nodules en fonction des doses de molybdène quelle que soit la période de fauche. De même, aucune relation n'a pu être établie entre les nombres de nodules et les périodes de fauche. 
Tableau 1 : Evolution de la biomasse foliaire (tMS/ha) de Desmodium intortum en fonction des doses croissantes de molybdène aux différentes périodes de fauche.

\begin{tabular}{ccccccc}
\hline Traitements & \multicolumn{5}{c}{ Périodes de fauche (jours) } \\
\cline { 2 - 6 }$(\mathbf{k g M o} / \mathbf{h a})$ & $\mathbf{3 0}$ & $\mathbf{6 0}$ & $\mathbf{9 0}$ & $\mathbf{1 2 0}$ & $\mathbf{1 5 0}$ & $\mathbf{1 8 0}$ \\
\hline 0 & $2,27^{\mathrm{a}}$ & $2,82^{\mathrm{b}}$ & 25,1 & 23,7 & 18,6 & 12,4 \\
0,75 & $2,16^{\mathrm{ab}}$ & $5,69^{\mathrm{ab}}$ & 15,9 & 14,4 & 15,1 & 16,3 \\
1,5 & $1,07^{\mathrm{b}}$ & $6,39^{\mathrm{a}}$ & 19,6 & 18,6 & 21,8 & 10,4 \\
2,25 & $2,01^{\mathrm{ab}}$ & $4,95^{\mathrm{ab}}$ & 13,8 & 14,5 & 10,1 & 17,8 \\
SEM & 0,032 & 0,053 & 0,070 & 0,078 & 0,096 & 0,038 \\
Prob. & 0,11 & 0,13 & 0,71 & 0,73 & 0,39 & 0,13 \\
\hline
\end{tabular}

Les moyennes portant les mêmes lettres dans la même colonne ne sont pas significativement différentes au seuil de 5\%. SEM : Standard Error of Mean; Prob.: probabilité

Tableau 2 : Evolution de la biomasse nodulaire (kg MS/ha) de Desmodium intortum aux différentes périodes de fauche en fonction des doses de fertilisation.

\begin{tabular}{ccccccc}
\hline Traitements & \multicolumn{7}{c}{ Périodes de fauche (jours) } \\
\cline { 2 - 7 }$(\mathbf{k g M o} / \mathbf{h a})$ & $\mathbf{3 0}$ & $\mathbf{6 0}$ & $\mathbf{9 0}$ & $\mathbf{1 2 0}$ & $\mathbf{1 5 0}$ & $\mathbf{1 8 0}$ \\
\hline 0 & 47,9 & 114 & 209 & 108 & 115 & 61,7 \\
0,75 & 50,6 & 120 & 134 & 148 & 102 & 25,2 \\
1,5 & 9,73 & 90,2 & 194 & 177 & 118 & 45,6 \\
2,25 & 36,8 & 85,2 & 280 & 136 & 94,9 & 45,3 \\
SEM & 0,13 & 0,043 & 0,061 & 0,11 & 0,10 & 0,095 \\
Prob. & 0,15 & 0,71 & 0,39 & 0,72 & 0,81 & 0,38 \\
\hline SEM : Standard Error of Mean; Prob.: probabilité.
\end{tabular}

Tableau 3 : Evolution du nombre de nodules de Desmodium intortum aux différentes périodes de fauche en fonction des doses de fertilisation du molybdène.

\begin{tabular}{ccccccc}
\hline Traitements & \multicolumn{7}{c}{ Périodes de fauche (jours) } \\
\cline { 2 - 6 } (kgMo/ha) & $\mathbf{3 0}$ & $\mathbf{6 0}$ & $\mathbf{9 0}$ & $\mathbf{1 2 0}$ & $\mathbf{1 5 0}$ & $\mathbf{1 8 0}$ \\
\hline 0 & 139 & 262 & 298 & 257 & 239 & 149 \\
0,75 & 163 & 193 & 338 & 187 & 160 & 45,3 \\
1,5 & 58,3 & 197 & 280 & 304 & 225 & 86 \\
2,25 & 160 & 148 & 328 & 267 & 98,3 & 52,7 \\
SEM & 0,095 & 0,05 & 0,037 & 0,065 & 0,084 & 0,097 \\
Prob. & 0,24 & 0,56 & 0,96 & 0,56 & 0,32 & 0,27 \\
\hline
\end{tabular}

SEM : Standard Error of Mean; Prob.: probabilité. 


\section{DISCUSSION}

Des résultats obtenus de cette étude, il apparait que la fertilisation au molybdène a influencé de manière variable la biomasse foliaire de Desmodium intortum en fonction des périodes de fauche. La baisse de biomasse foliaire avec les doses croissantes de molybdène observée à la première période de fauche est contraire aux résultats de Brkić et al. (2004) qui ont constaté une augmentation de la biomasse aérienne avec la fertilisation au molybdène mais en présence d'azote sur le pois chiche (Cicer arietinum). Cette différence pourrait donc être attribuée à la présence de l'azote, facteur essentiel de la croissance des plantes qui aurait pu induire une accélération de la croissance dès la mise en place. Au $60^{\mathrm{e}}$ jour de fauche, les valeurs de biomasses ont augmenté avec les doses croissantes de molybdène jusqu'à la dose de $1,5 \mathrm{~kg} \mathrm{Mo} / \mathrm{ha}$ avant de baisser avec la dose de 2,25 kg Mo/ha. Cela pourrait suggérer que la dose de $1,5 \mathrm{~kg} \mathrm{Mo} / \mathrm{ha}$ est dans les conditions de notre étude, la dose optimale de fertilisation. Au delà, le molybdène devient phytotoxique. Cette observation ne concorde pas avec celle faite par Odet et al. (1989) qui ont remarqué la phytotoxicité au-delà de $2 \mathrm{~kg}$ $\mathrm{Mo} / \mathrm{ha}$; à moins que la teneur en Mo du sol ne se soit ajoutée à la dose apportée pour affecter négativement cette biomasse dès ce niveau de fertilisation. Les valeurs de biomasse les plus élevées ont été observées à partir du $60^{\mathrm{e}}$ jour de fauche. Cette augmentation pourrait s'expliquer par le développement optimal des nodules (fixation optimale de l'azote) ayant induit une croissance importante de la plante. La relation hautement significative $(p<0,01)$ établie entre les biomasses foliaires et les périodes de fauche montrerait que la variation de la biomasse foliaire peut être expliquée dans $51 \%$ des cas par la période de fauche.

$\mathrm{Au}$ niveau des nodules, la fertilisation aux différentes doses de molybdène n'a pas eu d'effet significatif pour l'ensemble des périodes de fauche. Ce résultat corrobore celui de Jongruaysup et al. (1993) qui ont remarqué que la présence du molybdène n'a pas d'effet significatif sur l'initiation et le développement des nodules de Vigna mungo L. Dans l'ensemble des périodes de fauche, les valeurs les plus élevées de biomasses nodulaires ont été enregistrées au $90^{\mathrm{e}}$ jour correspondant ainsi au pic de nodulation dans le temps pour cette espèce, dans les conditions de notre essai. Ces valeurs sont largement au dessus de celle observée $(86,7 \mathrm{kgMS} / \mathrm{ha})$ par L'taief et al. (2009) sur le pois chiche en saison pluvieuse. Cette différence pourrait être attribuée à l'espèce. Les biomasses nodulaires les plus faibles ont été obtenues des plantes fauchées á la première et dernière période. Cette situation pourrait s'expliquer par la présence dans le sol de nitrate suffisant, au moment de la croissance pour inhiber la formation et la croissance des nodules (L'Taief et al., 2009) et de la sénescence des nodules qui intervient dans le temps (Trinchant et al., 1997).

La relation établie entre la biomasse nodulaire et la période de fauche qui se traduit par un coefficient de détermination relativement élevé $\left(\mathrm{R}^{2}=0,76\right)$ signifierait que dans $76 \%$ des cas, les variations de la biomasse nodulaire pourrait s'expliquer par période de fauche. Par contre, la relation entre la biomasse nodulaire et la biomasse foliaire s'est traduit par un coefficient de détermination faible $\left(\mathrm{R}^{2}=0,29\right)$. Ce qui semblerait montrer que la biomasse nodulaire ne saurait être un indicateur prédictible de la biomasse foliaire.

La fertilisation aux différents niveaux de molybdène n'a pas également influencé de manière significative le nombre des nodules quelle que soit la période de fauche considérée. Ce résultat est en accord avec Ceux observés par Brkić et al. (2004) sur le pois chiche (Cicer arietinum) selon lesquels la fertilisation au molybdène n'a pas eu d'effet sur le nombre de nodule et que les valeurs les plus élevées ont même été réalisées dans des variantes de production sans molybdène. L'évolution non régulière du nombre des 
nodules observée dans l'ensemble des périodes de fauche avec les niveaux croissants de molybdène ne corrobore pas les investigations de Jongruaysup et al. (1993) qui ont déclaré que le nombre des nodules baisse avec l'application du molybdène.

Indépendamment des niveaux de fertilisation au molybdène, les nombres des nodules ont de manière générale augmenté avec les périodes de fauche, avant de baisser à certaines périodes suite à la sénescence de nodules réduisant de ce fait le nombre.

L'absence de relation entre le nombre des nodules et les périodes de fauche observée dans cette étude, pourrait s'expliquer par la durée d'activité relativement brève des nodules, de l'ordre de quelques semaines chez les légumineuses herbacées. Cette courte période d'activité est compensée par l'apparition de générations successives de nodosités (Trinchant et al., 1997).

\section{Conclusion}

De l'étude de l'effet de la fertilisation au molybdène sur les biomasses et le nombre de nodules de Desmodium intortum dans l'Ouest Cameroun, il ressort que :

- la fertilisation au molybdène a influencé de manière variable la biomasse foliaire de Desmodium intortum en fonction des périodes de fauche. Les valeurs de biomasse les plus élevées ont été observées à partir du $60^{\mathrm{e}}$ jour de fauche, période au cours de laquelle les nodules atteignent la maturité et commencent le processus de fixation de l'azote ;

- la fertilisation aux différentes doses de molybdène n'a pas eu d'effet significatif pour l'ensemble des périodes de fauche sur la biomasse nodulaire ;

- la fertilisation aux différents niveaux de molybdène n'a pas également influencé de manière significative le nombre des nodules quelle que soit la période de fauche considérée.

- Dans l'ensemble, les biomasses et le nombre des nodules les plus élevés ont été observés à la période de fauche de 90 jours.

\section{REMERCIEMENTS}

Les auteurs remercient le Programme d'Appui Institutionnel-Développement des Ressources Humaines (PAI-DRH) du Ministère du Budget, des Comptes Publics, de la Fonction Publique et de la Reforme Administrative de la République Gabonaise pour le financement apporté à ces travaux.

\section{REFERENCES}

Bambara S, Ndakidemi PA. 2009. Effect of Rhizobuim inoculation, lime and molybdenum on photosynthesis and chlorophyll content of Phaseolus vulgaris L. African Journal of Microbiology Research, 3(11): 791-798.

Berger M, Belem PC, Dakouo D, Hien V. 1987. Le maintien de la fertilité dans l'Ouest du Burkina Faso et la nécessite de l'association agriculture-élevage. Coton et Fibres tropicaux, XLII(3): 10-13.

Bhupal GR, Patnaik MC, Singh MV, Khadke KM, Jagannadham. 2009. Effect of application of Molybdenum in maizeniger cropping System grown on acid soils of high altitude zone of Andhra in India. The proceeding of the International plant Nutrition Colloquium XVI, Department of Plant Sciences, UC Davis.

Brkić S, Milaković Z, Kristek A, Antunović M. 2004. Pea yield and its quality depending on inoculation, nitrogen and molybdenum fertilization. Plant, Soil and Environment, 39(1): 39-45.

Gomez KA, Gomez AA. 1984. Statistical Procedures for Agricultural Research ( $2^{\text {nd }}$ edn). Awiley InterScience Publication, John Wiley \& Son: Singapore; 680.

Jongruaysup S, O'Hara GW, Dell B, Bell RW. 1993. Effet de la fertilisation au molybdène afaible doses sur l'initiation des nodules, le développement et la fixation de $\mathrm{N}_{2}$ sur noir gramme (vigna mungo L.). Plant and soil, 155-156(1): 345-348. 
Le soudeur C, Labreuche J. 2007. Implanter une légumineuse à l'interculture. Perspectives agricoles. http://www. agriculture-de-conservation.com/IMG/ pdf/PA-2007-04-legCI-pdf

L'taief B, Sifi B, Zaman-Allah M, Hajji M, Lachaal M. 2009. Effet de la fertilisation azotée, de l'inoculation par Rhizobium $s p$ et du régime des pluies sur la production de la biomasse et la teneur en azote du pois chiche. Biotechnol. Agron. Soc. Environ., 13(4): 537-544.

Morot-Gaudry JF. 1997. Assimilation de l'azote chez les plantes. Aspects physiologique, biochimique et moléculaire. INRA, 422p.

Odet J, Musard M, Wacquant C. 1989. Mémento fertilisation des cultures légumières. Ouvrage-Article. CTIFL(Paris-France), 398p.

Rakotoarison BR. 2005. Etude de la valeur nutritive de Desmodium uncinatum, Hedychium coronarium et Musa paradisiaca pour une meilleur valorisation des ressources fourragères des hautes terres Malgaches. Thèse de Doctorat, Ecole Supérieure Agronomiques de l'Université d'Antananarivo, 111p
Sapolin S. 2007. Un nouveau mécanisme symbiotique plante-bactérie prometteur pour l'agronomie. Actualité Scientifique. Fiche $n^{\circ} 269$. Institut de Recherche pour le Développement (IRD). Paris/France. www.ird.fr

Steel RG, Torrie JH. 1980. Principles and Procedures of Statistics. McGraw Hill Book C: New York; 633p.

Toure A. 2001. Etude comparée de l'influence de quatre plantes de couverture sur la flore et la Végétation des parcelles en jachère à la station de coton du CNRA de Bouake (Cote d'Ivoire). Mémoire de DEA de Botanique, Option Ecologie Végétale. Université de Cocody Abidjan, UFR Biosciences, 78

Trinchant JC, Drevon JJ, Rigaud J. 1997. Fixation symbiotique de l'azote. In Assimilation de l'Azote chez les Plantes. Aspects Physiologiques, Biochimiques et Moléculaires, Morot-Gaudry JF (ed). INRA Editions; 422p. 